\title{
Factores de éxito en el relevo generacional en empresas de familia: estudio de caso ${ }^{1}$
} Success factors in the generational change in family firms: A case study

Recibido: 1 de noviembre de 2012
Evaluado: 12 de diciembre de 2012
Aceptado: 20 de diciembre de 2012

\author{
Xiomara Cervera Galindo² (Colombia) \\ xcervera@unal.edu.co \\ Universidad Nacional de Colombia \\ Iván Alonso Montoya R. ${ }^{3}$ (Colombia) \\ iamontoyar@unal.edu.co \\ Universidad Nacional de Colombia \\ Luz Alexandra Montoya R. ${ }^{4}$ (Colombia) \\ lamontoyar@unal.edu.co \\ Universidad Nacional de Colombia
}

\section{Resumen}

El presente artículo relaciona algunos de los principales factores que contribuyeron al relevo generacional exitoso de la primera a la segunda generación de una empresa familiar en Bogotá. Además, se presenta un breve marco conceptual, su importancia y su participación en el mercado colombiano. En él se pone de manifiesto la importancia del

\section{Abstract}

This article lists some of the main factors contributing to the successful generational change from first to second generation of a family business in Bogota. It is also presented a brief conceptual framework, its importance and its involvement in the Colombian market. It highlights the important role of the predecessor, successor and the family,

1 Investigación realizada en la Maestría en Administración, dentro de la Línea de investigación en mercadeo, innovación y competitividad en gestión agropecuaria, MILAGRO.

2 Administradora de Empresas, candidata a Magíster en Administración, Universidad Nacional de Colombia, Bogotá.

3 Profesor asociado, Facultad de Minas, Universidad Nacional de Colombia, sede Medellín, carrera 80 N. 65 -223, Núcleo Robledo, Colombia, tel. (+574) 43090 00, correo: iamontoyar@unal.edu.co, Grupo de Investigación en Mercadeo, Innovación y Competitividad en Gestión Agropecuaria (Milagro).

4 Profesora asociada, coordinadora nacional del Concurso Docente, carrera 45 N.o 26-85, edificio Uriel Gutiérrez, Bogotá, Colombia, tel: (+571) 31650 00, ext. 18364, correo: lamontoyar@unal.edu.co, Grupo de Investigación en Mercadeo, Innovación y Competitividad en Gestión Agropecuaria (Milagro). 
papel del predecesor, el sucesor y la familia, la relación entre los factores familiares y la planeación de la sucesión, así como la relación entre esta y los resultados de la sucesión. Finalmente, se sugieren acciones y factores específicos para preparar su sucesión.

Palabras clave: empresa de familia, sucesión, liderazgo, éxito, planeación. the relationship between family factors and succession planning, as well as between it and the results of the succession. Finally, specific actions and factors are suggested to prepare a succession process.

Keywords: family business, succession, leadership, success, planning. 


\section{Introducción}

La familia es una institución fuerte, que se ha consolidado a través del desarrollo de la vida social humana. Mas allá de una simple unión de individuos con un vínculo temporal o circunstancial es una comunidad a la que se está ligado por toda la vida por un lazo emocional y no contractual que es indeleble, que no puede deshacerse por conveniencia.

Es el ejemplo más básico y perfecto del funcionamiento organizacional: [e]l ingreso proviene del ofrecimiento de un servicio o venta de un producto, existen gastos fijos y variables, se poseen recursos humanos emergentes (los hijos) a quienes hay que adiestrar y capacitar para que ocupen posiciones de importancia y cuyas decisiones permitan establecer direcciones en el futuro de la organización, claro, todo ello en un escenario ideal (Socorro, 2008, p. 44).

Por esto, el estudio de la empresa familiar (EF) está lleno de desafíos conceptuales. Algunos autores toman como referencia para estudiar la EF aspectos, como su tamaño, el grado de control en la propiedad que una o varias familias tengan sobre ella, la participación de la familia en la administración y dirección del negocio, el desarrollo gerencial y tecnológico y el grado de formalización de sus sistemas, el mayor o menor grado de profesionalización gerencial, el ámbito geográfico regional, nacional o internacional en que actúe y el traslapo entre generaciones de padres, hijos y nietos, entre otros aspectos (Romero, 2006).

Para Gersick, Davis, McCollom y Lansberg (1997), una EF es aquella organización de negocios en la cual la propiedad de los medios instrumentales o la dirección se hallan, operativamente, en manos de un grupo humano entre cuyos miembros existe relación familiar.

También, y de un modo general, se acepta que una EF es una organización controlada (propiedad) por una familia, donde dos o más miembros de ella trabajan, activamente, en esa organización (Belausteguigoitia, 2004), definiciones que demandan aclarar la dimensión de la propiedad, entendida en el sentido de que una parte suficiente de ella debe ser poseída por la familia; lo mismo sucede sobre el ejercicio del poder, pues, alguno o varios de los propietarios dedicarán la totalidad o una parte importante de su tiempo a trabajar habitualmente en la EF, ya como directivos o en calidad de miembros de un consejo de administración. Desde la continuidad, se requiere que, al menos, esté en el ejercicio gerencial una segunda generación como señal de transmisión exitosa de la empresa hacia las generaciones futuras, como testimonio de una práctica de valores de la familia en la empresa (Fundes, 2008).

Para el presente documento, se señalará una EF como aquella en la cual la propiedad y las decisiones están dominadas por los miembros de un grupo de afinidad afectiva (Casrud y Brannback, 2007). En consecuencia, las EF contravienen el principio de los modelos contemporáneos de organización: la separación de la propiedad de la administración (Daily y Dollinger, 1991). Esto da lugar a características individuales en una serie de diferencias de funcionamiento entre las EF y las empresas convencionales. Las empresas familiares no son iguales; cada una de ellas cuenta con características propias que se originan de las singulares dinámicas familiares (Gómez-Betancourt, 2005) y, en mayor medida, del 
medio cultural de cada país. Las diferencias en la cultura, la legislación, las tendencias hacen que en el mundo las empresas familiares no se comporten igual. La EF está organizada como propiedad individual, sociedad, compañía propietaria o como sociedad anónima en la que una familia posee acciones suficientes para mantener un interés mayoritario. Su característica distintiva es que una sola familia es su propietaria y administradora, por lo que es una organización comercial muy singular que integra dos sistemas diferentes: el comercial y el familiar (Lea, 1993). Los procesos de sucesión han sido determinados como factores de éxito en el mantenimiento organizacional de la empresa familiar; por eso, es tan importante el estudio de la delegación de poder para encontrar elementos fundamentales que permitan la permanencia de las empresas de familia.

Para el desarrollo de este documento, se tiene como pregunta central de investigación: ¿cuáles son los factores de éxito en un proceso de sucesión? Esta pregunta será desarrollada a través de la metodología de estudio de caso, la cual será resuelta a partir de un enfoque deductivo en el que se describirá el concepto de empresas de familia, los factores de sucesión y las empresas de familia en el caso colombiano. Posteriormente, se señalará el análisis de la empresa Miratex y se evaluarán los factores críticos de éxito sobre los cuales se considera que la empresa ha logrado solventar sus dificultades.

\section{La empresa familiar}

El estudio de la EF como sistema nació con unos artículos independientes publicados en las décadas de 1960 y 1970. Los artículos se centraban en problemas comunes que parecían entorpecer a las EF: nepotismo, rivalidad entre generaciones y hermanos, gerentes no profesionales, entre otros (Gersick y et al., 1997). El modelo conceptual sostenía que la EF constaba, en realidad, de dos subsistemas interconexos: la familia y el negocio (Becknard y Dyer, 1983). Cada uno de esos dos círculos tiene sus propias normas, reglas de pertenencia, estructura de valores y organizaciones. Los problemas surgen porque los mismos individuos han de cumplir obligaciones en los dos círculos; por ejemplo como padres y como gerentes. Además, la empresa debe operar siguiendo las prácticas y principios establecidos, pero sin dejar de atender las necesidades del empleo, la identidad y los ingresos de la familia.

Durante los últimos años, se ha producido un creciente interés en el estudio de la EF, de esta manera se ha abordado la problemática desde múltiples puntos de vista: terapia familiar, psicología clínica, sociología, administración de empresa, historia y economía (Dyer, 1994). No se trata de un interés exclusivo creado por los académicos, también los empresarios, los profesionales e incluso los medios de comunicación comienzan a percatarse de que la EF significa mucho más que la tienda de la esquina (Aronoff y Ward, 1991) y a reconocer la importancia de este tipo de organizaciones para el tejido empresarial de cualquier país del mundo (Dyer, 1994). Este interés se debe, principalmente, a que la proporción de empresas de todo el mundo cuya propiedad o dirección se encuentra en manos familiares está entre 65 y $80 \%$ (Gersick y et al., 1997). Lo que realmente hace que una empresa sea familiar es la existencia actual, y el deseo de que 
continúe existiendo en el futuro, de un importante nexo de unión entre empresa y una familia. Cuando ese nexo está lo suficientemente fortalecido y se incorporan la cultura, formada por los supuestos básicos de actuación y por los valores, es permanente y voluntariamente compartida y pasa de generación en generación (Gallo, 1997). En las empresas familiares, las relaciones interpersonales de los miembros de la familia propietaria y las acciones que ocurren en la empresa se afectan mutuamente. Las consecuencias de los cursos de acción que se toman en el ámbito familiar suelen trascenderlo y extenderse hacia la dinámica de la empresa, por lo que el manejo de las fuerzas de la EF (empresa, familia y propiedad) es un factor clave no solo para una gestión exitosa de esta a corto y mediano plazo, sino también a largo plazo, en el que una sucesión exitosa puede determinar el rumbo de la empresa en situaciones que pueden tener en un extremo su consolidación y en otro su desaparición. Generalmente, el gerente fundador de una EF tiene una fuerza dominante que mantiene en equilibrio, aunque sea precario, entre las acciones en la empresa y la vida familiar, que se ve alterado cuando llega el momento en que cede el control de la empresa a otro miembro de la familia.

A menudo, las consecuencias son graves para las empresas. Un análisis realizado por Lea (1993) sobre una EF en un país latinoamericano mostró que solo 30 $\%$ de las empresas familiares logran una transición efectiva, mientras que $70 \%$ restante se retira del negocio junto con sus fundadores. Los resultados del conocido estudio del Wharton Entrepreneurial Center, aunque no estuvo específicamente enfocado al proceso de la sucesión, dio buena cuenta de su importancia evidenciando en 1984 que de 200 empresas familiares que eran exitosas en 1924 , $80 \%$ había dejado de existir y solo 20 $\%$ sobrevivía con el mismo nombre. Este porcentaje se integraba por $5 \%$ que habían sido vendido, $2 \%$ que no estaba en manos familiares y $13 \%$ que pertenecía a la misma familia que en 1924 . Este 13 $\%$ se integraba por $3 \%$ que había crecido notablemente, $3 \%$ que no había crecido y $7 \%$ que había declinado en varios sentidos (Wharton Entrepreneurial Center, 1984). Sumándose a los anteriores análisis, el Centro de Innovación y Desarrollo Empresarial sostiene que $67 \%$ de las empresas familiares no pasan a la segunda generación y $85 \%$ desaparece antes de la tercera generación (Navactiva, 2003).

Las cifras anteriores muestran que la nueva generación de gerentes no siempre robustece los éxitos de sus antecesores, posiblemente, a causa de la falta de habilidad estratégica, esfuerzo o compromiso de las nuevas generaciones o a la fugacidad de las oportunidades del mercado que favorecieron el éxito de las empresas. Puesto que de la actuación de los gerentes depende de la elección de los sus sucesores y su formación $y$, a su vez, de esta dependen las competencias del gerente sucesor para la identificación y aprovechamiento de las nuevas oportunidades de mercado, surge la necesidad de indagar la posible intensidad y sentido de la relación entre el perfil gerencial del antecesor y la planeación de la sucesión con respecto a los resultados de la sucesión, que se reflejan en la rentabilidad de las empresas y la satisfacción de la familia propietaria con estos resultados (Levinson, 1981).

Además de los conflictos clásicos de empresa, originados por la selección adversa o el riesgo moral, Schulze, Lubatkin, Dino y Buchholtz (2001) señalan 
un conflicto para el caso particular de la empresa familiar: el altruismo asimétrico. Este se manifiesta como un problema de oportunismo derivado de la visión distorsionada (y normalmente benévola) que unos miembros de la familia (padres) pueden tener sobre otros (hijos) respecto de su rendimiento, debido a la relación de parentesco que los une.

La identificación de los factores clave para que los sucesores gerenciales generen buenos resultados de la sucesión para la empresa y la familia es relevante para orientar los procesos relacionados con la sucesión en beneficio de los propios directivos, tanto de los antecesores como de los sucesores, así como de los miembros de las familias propietarias de las empresas y de las propias empresas, cuyo peso conjunto es importante en las economías.

\section{Empresa de familia en Colombia}

Hoy en día $70 \%$ de las empresas en Colombia son empresas de familia (Superintendencia de Sociedades, 2006). En un estudio elaborado por la Superintendencia en 2001, se realizó una muestra de $600 \mathrm{EF}$ en Colombia, cuyos resultados arrojaron que $33.9 \%$ de estas están aún en poder del fundador, es decir, no han afrontado un proceso de sucesión, 29.7 $\%$ está en poder de los hijos, es decir, en la segunda generación, y solamente $3.4 \%$ está en manos de la tercera generación (p. 125). Estas cifras motivan a pensar que la gran mayoría de las empresas familiares en Colombia puede estar en una etapa del proceso de sucesión, entre la primera y segunda generación, lo cual reviste un momento riesgoso para su continuidad si no se toman las medidas necesarias para su preparación (Fundes, 2008). Teniendo en cuenta que las EF representan casi 60 $\%$ del producto interno bruto [PIB ]del país, es necesario hacer un estudio que permita ofrecer variables que soporten la toma de decisiones para su supervivencia, así como para contribuir a mejoras en su gestión, las cuales les permitan crear una ventaja competitiva y, de esta manera, lograr un posicionamiento en el mercado (Gómez F., 2007).

Entre las corrientes de pensamiento sobre el tema hay diferencias, pues, algunos le dan mayor importancia al aspecto familiar que al empresarial, mientras que otros se concentran en los negocios. ¿Cuál es el más recomendable? Depende, fundamentalmente, de la tradición cultural y del tipo de familia. Por lo general, los anglosajones se preocupan más por el negocio, lo que facilita los procesos. Los latinos, por el contrario, valoran mucho las relaciones familiares y le dan una gran importancia a la continuidad de la familia en el tiempo, aunque esto implique llegar a decisiones que no son las mejores para el negocio (Gómez, 2007).

Resulta imprudente encarar el grave problema que suponen en toda Latinoamérica las empresas familiares si no se tiene a la vista la figura histórica de esa sociedad que hacía girar sobre el eje de la familia toda la actividad comercial, al [hasta el] punto [de] que la inversión, la producción y el consumo no tenían una necesidad interna de circulante (Instituto de Estudios Superiores de la Empresa [IESE], 1986, p. 62).

Efectivamente, las estrechas relaciones entre la agricultura y la familia se trasladaron con naturalidad al trabajo artesanal, lo que creó muchos de los rasgos característicos de las actuales empresas 
familiares. El origen de esas actividades agrícolas y artesanales tenía un carácter de familia patriarcal, que incluía hasta los parientes políticos, de segundo y tercer grado, y al que se le sumaban los ahijados, los empleados domésticos y hasta los asalariados de confianza. El vínculo familiar y el vínculo de trabajo se encuentran en esta figura social tan íntimamente unida que se configura en ella una grave confusión entre lo familiar y lo laboral. Los hijos llegan a tener un valor económico, pues, son considerados como ayudantes o jornaleros, es decir, mano de obra barata para ejecutar las actividades propias del negocio de la familia (IESE, 1986).

A pesar de tan lamentable confusión, que con mayor o menor intensidad aún hoy se presenta en las EF, esta extensión del ámbito familiar a las actividades laborales puede resultar hasta cierto punto beneficiosa. La familia constituye la unidad básica, estable y última de todo componente social: es el núcleo vital fundamental de toda sociedad. De ella surge como una extensión lógica la actividad política familiar, en ella nace el primitivo sentimiento religioso, así como las formas tempranas de actividades comerciales e industriales son más una prolongación del sistema familiar que una ruptura con él; más que una ruptura, se da, en rigor, un desdoblamiento (Dyer, 1994).

Colombia muestra una actividad económica apoyada en empresas de escala económica en crecimiento, donde las pymes, sumadas a las microempresas, representan $95 \%$ del parque empresarial del país y generan $63 \%$ del empleo nacional y $53 \%$ de la producción bruta de los sectores industrial, comercial y de servicios. De este grupo, se cuenta con una población de más de $68 \%$ de sociedades de familia, las cuales, en su gran mayoría, han tenido o tendrán que afrontar aspectos propios de su dinámica, como la sucesión del mando, el relevo generacional, la transición y evolución estratégica hacia un entorno cada vez más competitivo, donde elementos propios de la operación pueden incidir sobre la propiedad a través de capitalizaciones o inversiones de terceros para generar capital que fomente el crecimiento (Jaramillo, 2003).

Además, y como elemento asociado a la sucesión y según las estadísticas mundiales, menos de $13 \%$ de estas empresas logra sobrepasar a la tercera generación. En Estados Unidos, por ejemplo, y para hacer una comparación, los negocios de familia proporcionan $60 \%$ del empleo, $78 \%$ de todos los nuevos empleos, más de $50 \%$ del Producto Interno Bruto PIB y $65 \%$ de todos los salarios pagados. Por otra parte, en el Reino Unido los negocios de familia son más de $75 \%$ (Federación Nacional de Cultivadores de Palma de Aceite [Fedepalma], 2003) de todos los negocios organizados, y se estima que más de $50 \%$ de la gente empleada está contratada por negocios de familia. En América Latina, en México, por ejemplo, $56 \%$ de las ventas de las 100 mayores empresas son efectuadas por empresas de familia. En Venezuela la cifra es de 34 $\%$ (Fedepalma, 2003). Seguramente, si se hiciera un estudio similar en Colombia en estos días, los resultados darían cifras tan o más importantes que las mencionadas. La importancia de las anteriores afirmaciones en la economía del país se ve realzada cuando se observa que, en Colombia, la mayoría de estas empresas de familia $(72.8 \%)$ fueron fundadas después de 1970, lo cual indicaría que muchas de ellas se encuentran en una etapa de crecimiento y madurez, ávidas de 
recursos financieros (Vélez, 2005). Como los recursos internos de la familia propietaria pueden ser escasos, si estas empresas quieren continuar con su desarrollo, deben acudir a fuentes externas de financiación: préstamos o capital. Los préstamos bancarios no son fáciles de conseguir muchas veces, como lo revela el mismo estudio mencionado de la Superintendencia; y además, esos créditos bancarios son costosos, sobre todo, cuando se comparan con los costos de capital de las empresas que compiten con ellas a nivel internacional (Superintendencia de Sociedades, 2006). El aporte de capital de socios nacionales o del exterior, que sería la otra fuente de financiación para crecer, se vería frenada, entre otras cosas, por el sistema de gobierno al cual los inversionistas se refieren con insistencia. La resistencia que pueden presentar las empresas de familia a las reformas, que es común en la mayoría de los países emergentes, ha sido explícitamente esbozada en un reciente estudio de McKinsey y Company en el que establece que los gobiernos nacionales, como legisladores, deben reconocer la importancia de los negocios de familia en estos países, porque si no estas EF pueden generar verdaderos obstáculos a las reformas y su resultados no se verían a corto plazo. Continúa el estudio diciendo que, en muchos casos, las empresas se han preocupado más por dar cumplimiento a algunas de las reglas planteadas por la ley que realmente por realizar las mejoras necesarias en sus sistemas de gobierno (Elstrodt, H., 2003).

\section{La sucesión}

De acuerdo con Sharma (2001), la planificación de la sucesión es un factor importante en las EF por dos razones: en primer lugar, las actividades relacionadas con la planificación de la sucesión son parte del proceso; en segundo lugar, la planificación de la sucesión es reconocida como un medio para mejorar la tasa de éxito de la transición de la propiedad. La planificación de la sucesión tiene tres objetivos principales: 1) distribuir de manera eficiente y justa los activos a los jóvenes generaciones, 2) pasar el control del negocio de una manera que asegure el liderazgo efectivo del negocios y 3 ) promover y mantener la armonía familiar. A pesar de los muchos esfuerzos que se han hecho para alcanzar estos tres objetivos en las EF, no lograron un recurso efectivo para la sucesión, sino que, por el contrario, esto condujo a la agonía, la confusión y la parálisis (Davis, 1992). Sharma, Chrisman y Chua (1996) y Morris, Williams y Allen (1997) sugieren que los planes de la sucesión bien desarrollados pueden aumentar la probabilidad de la cooperación entre los interesados en la EF y, en la misma medida, aumentando la posibilidad de una sucesión fluida y eficaz. Sin embargo, a pesar de la importancia y la necesidad de la planificación, los empresarios y administradores rara vez planean el futuro de su sucesión (Sharma, Chrisman y Chua, 1996; Astrachan y Kolenko, 1994). De acuerdo con Lansberg (1988), la mayoría de los interesados en las EF son psicológicamente indiferentes a la planificación de la sucesión. Los fundadores de la compañía enfrentan factores psicológicos que les impiden la planificación de la sucesión, debido a que esto podría significar la pérdida paulatina del poder. Los miembros de la familia evitan la planificación, a causa de su preocupación por factores como la pérdida de identidad, armonía familiar y privacidad. Los altos directivos, que han 
trabajado una gran parte de tiempo con los titulares, son reacios a transferir desde una relación personal con el titular hasta una más formal con el sucesor. Los sucesores, por otra parte, se deben preparar para manejar los conflictos de esta naturaleza (Sharma, Chrisman y Chua, 1996; Kets de Vries, 1993).

La ausencia de un plan de sucesión puede causar graves problemas de gestión, incluso llevando a un fracaso en los negocios (File y Prince, 1996). Shulman (1991) considera que las EF deberían comenzar a pensar acerca de transferir la propiedad y la responsabilidad administrativa desde cinco hasta veinte años de anticipación, opinión también expresada por Dyck y Mauw (2002) y Davis (1992). En la mayoría de las EF, algunos de los altos cargos se heredan debido a que los familiares directos son conocidos por ser los sucesores desde el día en que nacen. La mayoría de las veces, y no importa qué tan alto es el profesional y de negocios, el sucesor suele ser el hijo del director general o el presidente. En ocasiones, puede estar preparado para ello. Es un riesgo al que la empresa debe someterse y puede llegar a tener resultados catastróficos (Domínguez, 2010). Es importante que los sucesores estén interesados en el proceso de sucesión. En ausencia de un sucesor que sea administrativa y físicamente capaz de hacerse cargo de la propiedad, la sucesión dentro de la familia rara vez se va a producir. Por lo tanto, el desarrollo del proceso del sucesor es de gran interés para investigadores externos y profesionales (Ibrahim y Dumas, 2001). Fiegener y Brown (1994) comparan el desarrollo de un sucesor en empresas de familia y en no familiares, y concluye que las EF son más personales, directas y enfocan las relaciones para el desarrollo del sucesor, mientras que las no familiares confían más en lo formal, individual y enfocado en tareas centrales. Lansberg (1998) sugiere que para ser efectivos tanto tutores como mayores deben entender la diferencia entre padres y mentores. La clave para una efectiva sucesión es encontrar una combinación oportuna entre los actores. En el conjunto, en un proceso de sucesión, para lograr un asesoramiento eficaz, los mayores deben negociar el proceso con sus mentores desde el principio, especificando las tareas y competencias que deben ser dominadas en cada etapa. De la misma manera, los mentores deben ir desarrollando actividades reales que les permitan obtener y mejorar su nivel de experiencia y conocimientos, así como su desempeńo, lo que lleva al aumento paulatino de su autoridad.

Lansberg (1998) argumenta que la formación del sucesor es un indicador del compromiso de la familia con el negocio y la calidad de la relación entre propietario-gerente, y el sucesor. Se concluye que el compromiso de la familia hacia el negocio está positivamente relacionado con el grado de formación sucesor, y que la calidad de la relación entre propietariogerente y sucesor está positivamente relacionada con la extensa formación del sucesor. Un estudio realizado por Goldberg (1996) muestra también que la eficacia del negocio está relacionada con el desarrollo del sucesor, demostrando que la efectividad de los sucesores que tenían más años de experiencia en el negocio era mayor que los sucesores que no la tenían.

Para González, Guzmán y Trujillo (2010), los argumentos en contra de la gestión por parte del fundador o los miembros de la familia del fundador sostienen que el administrador se selecciona entre un grupo restringido de personas, $y$ 
es posible que él o ella no posea la capacidad de gestión para dirigir la empresa, debido a no haber sido educado en un nivel adecuado para la posición y no tener las habilidades de gestión necesarias y la experiencia.

\section{Factores de éxito en la sucesión}

El hecho de que los investigadores no enfoquen el problema de la sucesión desde un mismo punto de vista provoca que exista falta de consistencia en cuestiones, tales como la consideración de un proceso sucesorio como exitoso. Así, y tal y como señalan Kesner y Sebora (1994), algunos autores definen este concepto como la ocupación de una vacante, otros definen el éxito como la ocurrencia de un mínimo de alteraciones organizativas a corto plazo y, para otros, viene dado por la reacción del mercado tras la sucesión. No obstante, en la literatura se encuentran autores, como Friedman (1986), para los que las medidas de resultado del proceso de la sucesión vendrían dadas por indicadores múltiples, tales como la evolución de la reputación de la empresa, el desempeño financiero, la rotación en los niveles directivos y la eficacia percibida del sistema de sucesión por parte de un informante clave de la organización. En lo que a la EF se refiere, gran parte de los autores que tratan la cuestión de la sucesión asocian el éxito con criterios generales, como la supervivencia o continuidad de la empresa o la permanencia de la empresa en manos de la familia. De hecho, se parte de la idea de que el sueño dorado de todo fundador es lograr que su empresa continúe en activo en manos de sus herederos (Danco, 1980, 1992; Nelton, 1986; Ward, 1987; Barach y Ganitsky, 1995; Poza, 1995).
La integración de la siguiente generación en la EF puede tener una importancia estratégica para este tipo de organizaciones hasta el punto de que la integración exitosa de nuevos miembros de la familia es para muchas EF un fin tan importante como los factores de beneficio, los nichos de mercado y otros determinantes de la política empresarial del negocio (Barach, Ganitsky, Carson, y Doochin, 1988). En este sentido, Longenecker y Schoen (1978) y Handler (1989) consideran que la sucesión se produce de manera efectiva cuando un miembro de la familia de la siguiente generación asume el rol de liderazgo, así como la posición de liderazgo (título de presidente). Asimismo, para Longenecker y Schoen (1978) debe ocurrir además que el sucesor sea relativamente autónomo en dicha función, particularmente, respecto de su relación con su predecesor/padre, y puesto que es difícil determinar cuándo puede considerarse maduro a un sucesor y qué variará de un individuo a otro, se considera que la séptima y última etapa o de sucesión madura comienza dos años después de la transferencia de la presidencia. Aronoff y Ward (1994) destacan también esta necesidad de autonomía cuando señalan que la sucesión efectiva implica un momento claramente señalado en el tiempo en el que el actual ocupante del puesto se retira de la escena permitiendo, por lo tanto, que el nuevo director general opere sin interferencias.

Otros autores consideran que una sucesión eficaz implica que se mantenga el control del capital en manos de la familia. Así Goldberg (1991), en la misma línea que Churchill y Hatten (1987), considera que una sucesión es eficaz cuando se produce la transferencia de la propiedad y el control de los derechos de propiedad 
desde el actual director general al sucesor, además de la transferencia del control sobre la dirección de operaciones y la dirección estratégica. Para estos dos últimos autores, lo más importante, para que una sucesión pueda ser considerada como exitosa, es que el sucesor tenga la habilidad necesaria para mantener saneada a la EF por medio de un crecimiento sostenido y de una rentabilidad continuada.

De lo anterior se deduce que no existe consenso sobre qué es lo que hay que tener en cuenta a la hora de evaluar un proceso sucesorio como exitoso o fracasado; de hecho, parece existir cierta confusión sobre lo que es una sucesión efectiva, es decir que se haya producido de hecho, y una sucesión eficaz o exitosa, es decir que tenga consecuencias positivas. Otra cuestión sería, entonces, delimitar para quién o para qué son esas consecuencias positivas. Así Dyer (1986) señala la dificultad para diferenciar una transición exitosa de otra que no lo es, ya que algunas empresas familiares pueden llevar a cabo un proceso sucesorio que sea un éxito desde el punto de vista del negocio, pero donde las relaciones familiares quedan, prácticamente, destruidas y, a la inversa, en otras ocasiones se mantiene la armonía dentro de la familia a expensas de la empresa, de ahí que este autor considere una transición como exitosa cuando tanto la familia como la empresa mantienen una relativa eficacia después de que la sucesión ha tenido lugar. En la misma línea, Friedman (1991) afirma que deberían tenerse en cuenta consideraciones tanto familiares como empresariales al hablar de eficacia de los procesos sucesorios. Ampliando este planteamiento, y con la finalidad de determinar los criterios para evaluar el éxito en la sucesión en la EF, al seguir a Kesner y Sebora (1994) cuando a la hora de considerar las consecuencias de un proceso de sucesión diferencian entre cuestiones de carácter organizativo y cuestiones relacionadas con los grupos de interés; en consecuencia, se distingue entre lo que se puede denominar criterios objetivos para la definición del éxito, que estarían relacionados con el efecto de la sucesión sobre la eficacia, los resultados y la estrategia organizativos, y los criterios que se consideran de tipo subjetivo, puesto que intentarían captar qué consecuencias ha tenido la sucesión sobre los distintos grupos de interés en una EF, ya sean estos los fundadores, sus esposas, los miembros de la familia de la siguiente generación, los directivos no familiares, otros empleados, etcétera.

\section{Factores objetivos}

Es muy habitual encontrar trabajos que al conceptualizar una sucesión como exitosa tienen en cuenta el hecho de que ella tenga efectos positivos sobre la eficacia o resultados de la empresa. Ahora bien, sobre la cuestión de cómo medir la eficacia organizativa ha existido y existe un considerable debate, pero, al mismo tiempo, y como señala Robinson (1982), las opiniones parecen coincidir en la consideración de la eficacia organizativa como un concepto multivariante, pudiéndose tomar como indicadores factores, tales como el crecimiento de las ventas (Dyer, 1986; Churchill y Hatten, 1987; Ward, 1987; Handler, 1989b; Lea, 1993), el crecimiento de los beneficios y de la rentabilidad (Dyer, 1986; Lea, 1993; Astrachan y Kolenko, 1994), los incrementos en la productividad (Lea, 1993) y la creación de empleo (Dyer, 1986; Astrachan y Kolenko, 1994). Este último criterio podría ser especialmente relevante, puesto que se ha 
atribuido a la EF un importante potencial para generar empleo (Shanker y Astrachan, 1996) y puede reflejar, en cierta medida, la dimensión social de la eficacia de la empresa.

\section{Factores subjetivos}

Cuando se considera la problemática de la EF, parece existir un consenso absoluto entre todos los autores en cuanto este tipo de empresas debe sus especiales características y, como consecuencia, sus ventajas e inconvenientes particulares, a la implicación en la gestión empresarial de una o de varias familias. Por consiguiente, la gestión de las EF se caracteriza por la introducción de elementos emocionales a la hora de tomar decisiones que pueden estar afectando la supervivencia futura de la empresa. Dada esta elevada implicación y compromiso de la familia en el negocio, las relaciones interpersonales e intergrupales en una EF tienen connotaciones particulares si se las compara con la gestión de otro tipo de organizaciones (Dunn, 1995; Gersick, Davis, McCollom y Lansberg, 1997), por lo que posible afirmar que existe un mayor número de elementos subjetivos en dichas relaciones. Por lo tanto, se entiende que no es suficiente limitar la consideración de éxito o fracaso de un proceso sucesorio al análisis de elementos puramente objetivos, tales como los mencionados más arriba, ya que el grado de satisfacción que experimenten determinadas personas o grupos de interés con él puede condicionar, en gran medida, el éxito futuro de la empresa.

En esta línea, Stempler define una sucesión exitosa como:
[...] aquella en que todas las partes implicadas en el proceso de sucesión perciben la sucesión como favorable y existe una satisfacción general de cada persona que participó en el proceso de transición. Además, no existe enfado u hostilidad de unas partes hacia otras. Existe también una percepción general de todos los agentes participantes en el proceso de que la familia, la empresa, los empleados no familiares, el sucedido y el sucesor fueron tratados de la mejor manera posible por el plan de sucesión (1988, p. 65).

Por su parte, Dyer (1986) afirma que es necesario tener en cuenta la buena marcha de la familia después de la transición, la cual puede determinarse utilizando criterios, como el grado de satisfacción de las necesidades y deseos de la familia, la habilidad para resolver los problemas $y$ amenazas a los que esta se enfrenta y el hecho de que las relaciones familiares sean satisfactorias. A este respecto, algunas de las preocupaciones que presentan los familiares, trabajen o no en la empre$\mathrm{sa}$, tienen que ver con sus ingresos, los conflictos familiares, las políticas de dividendos y el disponer de un lugar en la empresa para sus propios hijos (Barnes y Hershon, 1976).

Como se puede apreciar, es necesario considerar las perspectivas de los diversos implicados en el proceso que se pueden agrupar en las categorías siguientes: el sucesor, el predecesor, el cónyuge y los empleados no familiares (ver tabla 1). 
Tabla 1. Perspectivas de los diversos implicados en el proceso

\begin{tabular}{|c|c|}
\hline Factores objetivos & Factores subjetivos \\
\hline $\begin{array}{ll}\text { - } & \text { Posicionamiento } \\
\text { - } & \text { Permanencia } \\
\text { - } & \text { Crecimiento de las ventas } \\
\text { - } & \text { Crecimiento de los beneficios y de la rentabilidad } \\
\text { - } & \text { Incremento de la productividad } \\
\text { - } & \text { Ofrecimiento de empleo }\end{array}$ & $\begin{array}{l}\text { Relaciones interpersonales e intragrupales } \\
\text { Grado de satisfacción de las necesidades de la familia } \\
\text { Habilidades para resolver problemas y amenazas } \\
\text { Conflictos familiares } \\
\text { Políticas de dividendos } \\
\text { Disposición de incorporar familiares a la empresa }\end{array}$ \\
\hline
\end{tabular}

\section{El sucesor}

Handler (1989) orienta su investigación al estudio de la experiencia sucesoria del miembro de la familia de la siguiente generación que define como

[...] aquel individuo que es miembro de la familia que ostenta el control de la empresa (nuclear o extendida), que ha trabajado en la empresa en el pasado, que en la actualidad está trabajando en la empresa o que personalmente cree que lo estará en el futuro y que se percibe a sí mismo como un sucesor potencial de la siguiente generación al fundador o propietario (p. 259).

Se considera que uno de los resultados fundamentales del proceso sucesorio (variable dependiente) es la calidad de la experiencia de la sucesión, definida como el grado en que, desde la perspectiva del miembro de la familia de la siguiente generación, su implicación en la EF es satisfactoria y productiva. En tal sentido, el sucesor estará satisfecho con su experiencia en la medida en que pueda satisfacer sus necesidades profesionales, psicosociales, de etapa vital y de influencia personal; cuando se sienta implicado y comprometido con la empresa y experimente un sentido de propiedad, importancia y responsabilidad por las actividades que lleva a cabo en la organización, y en función de la calidad de las relaciones y dinámicas intergeneracionales e intrageneracionales. A su vez, otra variable que influye en la calidad de la experiencia de un individuo a lo largo del tiempo es la propia eficacia de la sucesión. Asimismo, esa calidad de la experiencia es crítica para la eficacia de la sucesión, que se mide respecto de los criterios de resultados organizativos (resultados financieros e iniciativa del miembro de la familia de la siguiente generación como sucesor sobre nuevas ideas, programas y productos), la satisfacción percibida de los miembros de la familia tanto dentro como fuera del negocio, el tiempo que el sucesor lleva en el cargo y el hecho de si se han transferido la presidencia, la responsabilidad y el capital de la empresa. Los resultados del estudio de Birley (1986) pueden apoyar esta idea de que los sucesores consideran su entrada en la EF como un medio de satisfacer necesidades e intereses personales y profesionales. Su trabajo, llevado a cabo con estudiantes universitarios de Administración de Empresas, concluye con la idea de que la decisión de los herederos sobre su incorporación a la EF está condicionada por su sentido de la responsabilidad hacia la familia y hacia la empresa y por el grado de interés en ella, que venía dado por aspectos relacionados con la empresa como entidad: sus objetivos, su orientación y su estrategia. La necesidad de que los implicados 
en la sucesión satisfagan sus necesidades suele ser causa de conflictos, sobre todo cuando, existen varios hermanos involucrados en el proceso de sucesión (Friedman, 1991; Swogger, 1991). Además, ocurren situaciones en las que el sucesor puede experimentar dificultades añadidas para conseguir una experiencia sucesoria satisfactoria; es el caso de que el sucesor sea un hijo menor o una hija. Los problemas adicionales se producen porque existe una incongruencia entre las jerarquías familiar y empresarial que puede llevar a que surjan cuestiones de ambivalencia, rivalidad, identidad y autoestima (Barnes, 1988).

Concretamente, la situación de las hijas sucesoras parece ser la más compleja, al enfrentarse a mayores dificultades para desarrollar su identidad, dados los niveles más altos de conflicto y ambigüedad de papeles que experimentan. A diferencia de los hijos varones, las hijas suelen entrar en la empresa sin papeles claramente definidos, tienden a ser invisibles como sucesores potenciales en la empresa y les cuesta más diferenciar su propia identidad de la de sus padres (Dumas, 1989, 1992). Sin embargo, diversos estudios como los de Dumas (1992), Frishkoff y Brown (1993) y Dumas, Dupuis, Richer y St-Cyr (1995) ponen de manifiesto que las capacidades y habilidades de las hijas son un importante recurso que, tradicionalmente, se ha sido infrautilizado en las empresas familiares, sobre todo, si se compara con los hijos varones. Así, las hijas, por los procesos de socialización a que son sometidas, son capaces de satisfacer sus propias necesidades y de establecer su propia identidad manteniendo una actitud de colaboración y de interdependencia con los padres, a diferencia de los hijos, que suelen adoptar actitudes de competencia e intentan establecer su independencia. De hecho, las hijas conceden mayor importancia a las relaciones interpersonales y se sienten motivadas por la posibilidad de equilibrar sus necesidades personales, familiares y profesionales en el contexto de la EF.

\section{El predecesor}

Desde el punto de vista del antecesor, la sucesión puede ser una cuestión con un gran componente emocional, en especial, si este es el fundador de la empresa. Parece ser que la continuidad empresarial es el sueño primordial de todo empresario familiar (Danco, 1980, 1992; Poza, 1995); y, sin embargo, su propia actitud hacia la sucesión puede llevarle, precisamente, a lo contrario, a lo que se ha dado en denominar eutanasia corporativa en referencia a "la acción del propietario que voluntariamente destruye la empresa que ama porque no es capaz de crear en vida una organización viable con una continuidad clara" (Danco, 1981, p. 75). Este mismo autor afirma que la actitud que puede proporcionar al predecesor un sentimiento positivo sobre la sucesión es la de considerar su retiro como un renacimiento, es decir,

[...] un período dorado en el que él [el propietario] dota a los que le siguen con la acumulación de conocimiento e inquietud que a él le ha costado toda una vida adquirir. A través de estos otros puede crear su inmortalidad (Danco, 1981, p. 110).

En lugar de enfrentarse al retiro de su empresa como el resultado de su propia muerte, puede vivir para ver la revitalización de su empresa a través del 
sucesor que él mismo ha elegido y formado (Danco, 1992). De acuerdo con Sonnenfeld (1988), esto podría implicar la adopción por parte del predecesor de un estilo de retiro del tipo embajador o gobernador. El primero se caracteriza porque, aun cuando el predecesor permanece en la empresa después de abandonar el puesto, no tiene intención de recuperarlo. Participan como asesores apoyando a sus sucesores. Esto tiene como consecuencia que experimenten la sucesión con sentimientos de logro y de aprecio, lo que, a su vez, origina que sus empresas se beneficien internamente por la transferencia de conocimientos, y de cara al exterior logran una apariencia de continuidad en el mando. Existen múltiples posibilidades para lograr estos beneficios, entre las cuales se cita la concesión al predecesor de un título emérito, asignarle las labores de relaciones públicas donde puede aprovechar el gran número de relaciones personales y profesionales que ha reunido a lo largo de su vida, asignarle un papel de consultor que permita seguir contando son su experiencia técnica o empresarial, servir como maestro de las nuevas generaciones o realizar tareas de análisis o planificación (Lea, 1993).

Por su parte, los predecesores que adoptan el estilo gobernador a la hora de retirarse tampoco se resisten a la sucesión, sino que, normalmente, dejan el puesto e inician una actividad completamente nueva. Este tipo de personas casi siempre ha cultivado otros intereses no relacionados con la empresa y el retiro para ellos no es más que un cambio de actividad que les sigue proporcionando un sentimiento de utilidad social. A pesar de las consecuencias positivas que tienen para la empresa y para él mismo la adopción de los dos estilos de retiro anteriores, la realidad parece indicar que, ante la sucesión, el fundador o dueño suele experimentar sentimientos de angustia ante la idea de la muerte, de pérdida de influencia y de identidad, así como una pérdida de una actividad significativa e incluso sentimientos de rivalidad y celos hacia el sucesor (Lansberg, 1988).

Debido a este alto arraigo que tiene el fundador o dueño de la EF con esta, la mayor parte de ellos adoptan un estilo del tipo monarca o general según la clasificación de Sonnenfeld (1988). Los primeros no abandonan su puesto a no ser que sean forzados por la muerte, por la mala salud o por una revuelta en palacio (renuncias de ejecutivos clave, ultimátums por parte de accionistas miembros de la familia o acciones por parte del consejo de administración), y los segundos tampoco abandonan el puesto hasta que se les echa y, cuando esto ocurre, hacen planes para regresar al poder, lo cual suele ocurrir ante una crisis real o supuesta que les proporciona la excusa de ser necesarios para la mejora de la situación ante la incompetencia del sucesor o sucesores.

\section{El cónyuge}

Normalmente, se trata de la esposa del fundador o dueńo. La importancia de su papel en la EF parece estar fuera de toda duda, y aun cuando su participación directa pueda no ser muy frecuente, todas las esposas están implicadas de manera importante en la actividad del negocio aunque solo sea por el extraordinario grado de apoyo que deben prestar a sus cónyuges (Danco, 1981; Kepner, 1983; Rosenblatt, De Mik, L., Anderson y Johnson, 1985; Gallo, 1990). Para los cónyuges, el proceso sucesorio puede ser la fuente de un complejo conjunto de retos, 
incertidumbres y preocupaciones acerca del futuro económico y emocional de la familia (Lansberg, 1988). Para ella, al igual que para el fundador, la empresa puede tener gran importancia como fuente de actividades y como componente de su identidad, $y$ al igual que este puede encontrar dificultades para enfrentarse a la sucesión. En ocasiones, el resultado de la sucesión es que son las propias esposas las que se convierten en sucesoras, sobre todo, tras la muerte inesperada del marido, que las obliga a hacerse cargo del negocio, ya sea para preservar la visión de su fundador, porque es la mejor forma de asegurar el futuro económico de la familia, las viudas pueden constituirse en una fuerza importante para la supervivencia y el crecimiento de las EF y, de hecho, las empresas, con frecuencia, florecen debido a que estas mujeres suelen estar abiertas a nuevas ideas y, a menudo, acaban dándose cuenta de que están dirigiendo la empresa porque obtienen satisfacción personal y no por sus maridos o incluso por sus hijos (Nelton, 1991).

\section{Los empleados no familiares}

Por su parte, los empleados y directivos que trabajan en la empresa, pero que no forman parte de la familia, tienen sus propias aspiraciones y motivaciones sobre el proceso de sucesión, en función de su historia personal (sobre todo, en la empresa), la etapa en la que se encuentran de su vida y su situación actual (en particular, en el aspecto laboral) (Davis, 1988). En este sentido, los empleados mayores están, básicamente, preocupados por la seguridad y quieren recompensas a su lealtad, participación en el capital y agradar al jefe, mientras que los jóvenes están más dispuestos a asumir riesgos y quieren profesionalismo, oportunidades para el crecimiento y razones que los motiven para quedarse (Barnes y Hershon, 1976). De hecho, los empleados con más antigüedad en la empresa y que han desarrollado una relación de tipo personal con el fundador pueden sentirse amenazados por el cambio, en el sentido de que esperan que la implantación de estructuras más formalizadas pueda suponer una restricción a su autonomía e influencia (Lansberg, 1988). Sin embargo, con frecuencia también ocurre que la continuidad de la familia proporciona seguridad y comodidad a los miembros ajenos a ella (Beckhard y Harris, 1988). Teniendo en cuenta todas estas consideraciones, los miembros ajenos a la familia van a adoptar una postura ante la sucesión que puede variar entre constituirse en aliados incondicionales del sucesor hasta sabotear por completo el proceso de sucesión, pasando por diversas posturas intermedias donde asumen papeles de asesores del sucesor, de puente entre dos generaciones en la EF, de evaluadores de los directivos familiares, de simples espectadores del proceso de sucesión e incluso de competidores por ocupar la dirección de la empresa.

\section{Liderazgo}

Debería ser uno de los principales requisitos del sucesor, de manera que le permita llegar a ser un líder exitoso. Para empezar, la innovación es una tarea importante que debe ser promovida por el líder. Existen dos principales tipos de líderes (Bass, 1997): los líderes transaccionales, que motivan a sus subordinados para llevar a cabo las actividades en los niveles esperados, ayudándolos a reconocer la responsabilidad de las tareas, identificar los objetivos, adquirir destrezas 
sobre el cumplimiento de los niveles de rendimiento, entender sus necesidades y cómo desean que las recompensas están relacionados con el logro de sus metas. Y, por otro lado, están los líderes de transformación, quienes motivan a los individuos a llevar a cabo sus actividades más allá de las expectativas normales, inspirándolos, de esta manera, a obtener un enfoque más amplio de las misiones, que van más allá de sus propios intereses, para concentrarse en un más alto nivel intrínseco en lugar de metas extrínsecas de nivel inferior, y tener confianza en sus habilidades para lograr las misiones extraordinarias articuladas por el líder. Según Bass (1985) son tres los factores más importantes que conducen a un liderazgo transformacional:

1) Carisma: este factor de liderazgo advierte que es el líder quien debe inspirar el orgullo, la fe y el respeto, y reconocer lo que es realmente importante. De igual manera, debe articular, efectivamente, el sentido de misión o visión, que inspira a sus seguidores.

2) Consideración individualizada: este factor de liderazgo implica proyectos de delegación para ayudar a desarrollar las capacidades de cada seguidor, prestando especial atención a las necesidades de los seguidores, y tratando a cada seguidor como una persona digna de respeto.

3) Estimulación intelectual: este factor de liderazgo consiste en ofrecer nuevas ideas para estimular a los seguidores para repensar viejas formas de hacer las cosas, impulsándolos a mirar los problemas desde múltiples puntos de vista y a fomentar avances creativos en los obstáculos que parecían insuperables.
Autores como Avolio y Bass (1991), Bass (1998) y Sosik (2002), partiendo de los tres factores de Bass (1985), incluían un cuarto factor adicional al que denominaron motivación inspiradora, al tiempo que el factor carisma empezaron a denominarlo influencia idealizada (Bass, 1998; Bass y Steidlmeier, 1999). La motivación inspiradora se refiere a la capacidad de los líderes transformacionales para generar entusiasmo, ánimo y optimismo, comunicando su visión de futuro alcanzable con fluidez y confianza, usando símbolos que orienten el esfuerzo de sus subordinados y consiguiendo de ellos los comportamientos deseados (Yukl, 2002). El líder transformacional motiva a sus seguidores a trabajar para conseguir objetivos trascendentes, en vez de objetivos egoístas a corto plazo, y para conseguir el autorreciclaje en lugar de seguridad; en este caso la recompensa para los seguidores es interna. Explicando su visión, el líder transformacional convence a sus seguidores para trabajar duro y conseguir las metas que tiene en su mente. Esta visión dota a los seguidores de una motivación para el trabajo que resulta ser autocompensatoria (Bass, 1999). Además, los líderes descritos como transformacionales o visionarios tienen efectos positivos sobre sus organizaciones y seguidores tanto en los resultados obtenidos por las organizaciones como en los niveles de satisfacción, compromiso e identificación (Fiol, Harris y House, 1999). Transforman las necesidades, valores, preferencias $\mathrm{y}$ aspiraciones de sus subordinados, consiguiendo que, en lugar de sus propios intereses, se esfuercen en pos de los intereses colectivos de la organización (Bass 1997; Kark, Shamir y Gilad, 2003).

Por su parte, los subordinados de este tipo de líderes se comprometen, 
firmemente, con la misión y los objetivos de la organización (Bass, 1988; Viator, 2001; Walumbwa, Lawler, Avolio, Wang y Shi, 2005). Teniendo en cuenta que, en el caso concreto de las EF, el liderazgo ejercido tiende a ser más transformacional que en las no familiares (Vallejo, 2003), los efectos positivos apuntados por la literatura, entre los que se encuentra el ya mencionado grado o nivel de compromiso con la organización cuyo estudio nos ocupa, han de tener también un mayor peso en este tipo de empresas. En este sentido, autores como Poza (1995), Aronoff y Ward (1994), Chami (1999) y Lee (2006) destacan la importancia y el peso específico en la cultura de la EF del valor del compromiso.

\section{Metodología de estudio}

Para la presente investigación, se utilizó la metodología de estudio de caso (Montoya y Montoya, A. y Montoya, I., 2010; Sharma, Chrisman y Chua, 1996), con el fin de analizar las competencias organizacionales utilizadas por una $\mathrm{EF}$, que fue seleccionada por cumplir con dos requisitos principales: fue fundada en Bogotá y cuenta con relevo generacional exitoso de primera a segunda generación. La metodología incluye una descripción de la empresa, historia y medición de variables a través de una entrevista a profundidad elaborada con el modelo de escala de Likert ${ }^{5}$ y encuesta cerrada, con el fin

5 Este método fue desarrollado por Rensis Likert hacia 1932; sin embargo, se trata de un enfoque vigente y bastante popularizado. Consiste en un conjunto de ítems presentados en forma de afirmaciones o juicios ante los cuales se pide la reacción de los sujetos. Es decir, se presenta cada afirmación y se pide al de identificar los factores exitosos más relevantes en la sucesión de empresas de familia de primera a segunda generación.

\section{Hipótesis}

H1: la relación de factores objetivos y subjetivos determinan el relevo generacional exitoso en la EF.

Hipótesis alterna: las empresas de familia dirigidas por sucesores que se han involucrado en su manejo desde temprana edad tienen más posibilidades de llevar a cabo un relevo generacional exitoso.

El proceso llevado a cabo para la consolidación de resultados permite una observación profunda a partir de las hipótesis descritas.

\section{Historia de Miratex}

Miratex fue fundada hacia 1948 en Bogotá por los hermanos Mishaan. Inician su negocio con la compra y venta de hilaza y la fabricación de telas. Posteriormente, y en vista del crecimiento del negocio, importaron hilanderías, combinando, de esta forma, la elaboración de hilazas y de telas. El patrimonio con el que iniciaron era muy pequeño. Las fábricas estaban en Bogotá, pero distribuían para todo el país. Desde muy temprano, Roy Mishaan, hijo de uno de los fundadores, tomó la iniciativa para conocer el negocio de la familia. Tenía siete años, y pasaba sus vacaciones entre hilazas y telas

\footnotetext{
sujeto que externe su reacción eligiendo uno de los cinco puntos de la escala. A cada punto se le asigna un valor numérico. Así, el sujeto obtiene una puntuación respecto de la afirmación y al final se obtiene su puntuación total sumando las puntuaciones obtenidas en relación con todas las afirmaciones.
} 
y limpiando conos. Ya cuando Roy Mishaan culminó sus estudios en Ingeniería Industrial conocía, perfectamente, el funcionamiento de la fábrica. Escogió esta carrera con la idea de desempeñarse polifacéticamente en la empresa, a pesar de que la carrera que al principio prefirió fue Arquitectura. Su hermano mayor estudió Ingeniería Textil y su hermano menor estudió Economía. En 1974 Roy Mishaan y su primo se incorporaron oficialmente a Miratex, luego de culminar sus estudios universitarios en el extranjero. Miratex pasa de mano de los padres a los hijos en 1987, quienes triplicaron su producción, abrieron una planta muy moderna en la avenida El Dorado (Bogotá) en 1993 e inauguraron una boutique en el norte de la ciudad. La distribución de los ingresos fue dividida por porcentajes, por grupo familiar y por socios. Actualmente, la familia de cada socio tiene participación en la empresa; es decir, cuñados, hermanos, primos y cada socio. Los primos Mishaan solicitaron un estudio de todos los negocios de la familia para determinar su valorización. Elaboraron un listado de propiedades para determinar qué le correspondía a cada quien, con el acompañamiento de un notario o un tercero imparcial, para que diera fe y quedara por escrito para futuras decisiones. Este proceso fue tedioso, extenuante y desgastador; duró un año y fue llevado a cabo en 2003. Finalmente, llegaron a varios acuerdos, y así es como en 2003, Roy Mishaan y su primo se separaron del negocio. Lo anterior debido al fallecimiento de su tío, uno de los fundadores. Posteriormente, y en ese mismo año, queda a la cabeza de Miratex Roy Mishaan y sus hermanos. Roy Mishaan manifiesta de la siguiente manera su percepción de las empresas de familia y de la industria textil:

Anteriormente, la ventaja de un negocio familiar (diferente a Fabricato y Coltejer) era que no había muchas decisiones que tomar, no había que contar muchos votos, sencillamente las decisiones las tomaba el gerente general y, de esta manera, el crecimiento era más rápido. Además, la industria textil para ese entonces estaba protegida. Hace treinta años había muchas restricciones para importar y los aranceles estaban alrededor de $35 \%$, lo cual hizo crecer no solo a la industria textil, sino a toda la industria colombiana. Luego llegó el presidente Gaviria, con su programa de apertura económica. La apertura es buena, pero siempre y cuando el país tenga la infraestructura para desarrollarla; Colombia no estaba preparada para la apertura. La industria textil desde entonces está desprotegida; la electricidad y el agua son de las más caras del mundo, la mano de obra es promedio, pero no es barata, los fletes terrestres son los más caros del mundo y es en uno de los pocos países en los que se tiene una tarifa mínima, no una máxima. Adicionalmente, la infraestructura vial que se derrumba cada dos meses hace todavía más difícil desarrollar la industria textil en Colombia. El Gobierno manifiesta que la industria colombiana no ha sido capaz de responder a una deman$\mathrm{da}, \mathrm{y}$ presenta una serie de ayudas, pero no se da cuenta de que existen demasiados factores que están afectando la industria y que no están siendo resueltos directamente. Lo valioso de una EF es que el corazón de la gente está metido allí. Cuando no hay corazón no hay dolientes y cuando no hay dolientes no hay 
quien detenga la liquidación de una empresa. Afortunadamente, para Miratex, existieron muchos dolientes que hicieron que se salvara.

Miratex lleva más de sesenta años de creada, hilando, tejiendo (mil toneladas de telas de punto y toalla) y confeccionando. Tanto es así que crearon un laboratorio que se encarga de crear nuevas texturas, nuevas telas y nuevas fibras. Estas últimas no se venden muy bien en el país, pero sí en el extranjero. De igual manera, transfirieron unas máquinas que en Venezuela producían $10 \mathrm{t}$ de toallas al mes y aquí las tienen produciendo $70 \mathrm{t}$ al mes. Se trata de reinventar, aprovechar y maximizar el negocio. El éxito de Miratex en los últimos tres o cuatro años ha sido la innovación en los productos y la elaboración de productos que no se pueden importar de otras partes: algodón con bambú, productos ecológicos y poliéster reciclado de botellas, entre otros.

\section{Resultados}

\section{Resolución de las hipótesis}

Frente a la primera hipótesis sobre la relación de factores objetivos y subjetivos que determinan el relevo generacional exitoso en una EF, se puede determinar que, al efectuar el análisis de los datos recolectados, a través de entrevista directa y de encuesta a través de variables, predominan más los factores subjetivos. En la continua búsqueda de estrategias para mantener la ventaja competitiva de la EF, el predecesor se vio impulsado a delegar responsabilidades y compartir el poder. Gradualmente, se ocupó de transmitir los valores y formas de trabajo a la generación siguiente, existiendo, a menudo, competencia para obtener los puestos de liderazgo entre los empleados y los miembros de la familia. Cuando el retiro del gerente se acercó, la distribución de la propiedad y las posiciones en la empresa se volvieron factores clave en la sucesión gerencial. Para Miratex, lo que empezó siendo una sociedad individual se transformó en la década de los ochenta en sociedad anónima. El proceso de sucesión a la segunda generación se encuentra culminado, y ya cuenta con sucesor en la presidencia, quien lleva trabajando oficialmente más de veinticinco ańos y le fueron concedidos poderes junto con algunos directivos miembros de la familia. Entre los factores subjetivos más importantes que se destacan en la sucesión están, entre otros, la presencia de liderazgo claro, la disposición a la comunicación y la concertación de criterios entre los miembros de la familia. Existe aquí un factor de análisis muy importante y es que la mayoría de las EF están en manos de sociedades entre hermanos. Las razones por las cuales se presenta esta situación son variadas, pero, de acuerdo con los datos analizados, prevalecen los lazos afectivos y de confianza que se sienten entre los hermanos que realizan esta alianza comercial. De igual manera, la razón final de tal comportamiento es la sucesión, donde tras el retiro de los fundadores que, generalmente, son los padres, son sus hijos, que a su vez son hermanos quienes a manera de herencia reciben la dirección y el manejo de las empresas de este tipo. También se encuentra como resultado que las sociedades entre padres e hijos se asocian por la confianza existente con la familia y por la falta de mecanismos que permiten garantizar los derechos de propiedad cuando la sociedad es no familiar, 
también, por la confianza que existe entre la misma familia por la relación familiar que se presenta en el interior del núcleo y por el deseo existente para que la empresa presente una continuidad hacia el futuro como propiedad de la familia. De igual manera, se evidenció la importancia del cónyuge en la conservación de la EF. El argumento principal se basa en que las EF que se encuentran en manos de matrimonios son matrimonios sólidos donde existe la confianza entre la pareja $y$ el deseo de que sus hijos sean los herederos de la empresa y puedan prolongarla hacia un futuro. También se encuentra que para la toma de esta decisión empresarial existe la firma de capitulaciones por anticipado por parte de las familias de los cónyuges $y$, por lo tanto, surge la necesidad de crear un patrimonio sólido que en un futuro les asegure a sus hijos la subsistencia. En este caso, los entrevistados argumentan ser matrimonios con varios años de convivencia donde existe una seguridad de permanencia, factor que señalan como importante, ya que es muy baja la probabilidad de separación gracias a la confianza que existe entre la pareja constituyente de la sociedad al ser conscientes de la amenaza existente para la supervivencia de la empresa el que un divorcio acerque la compañía al cierre. Generalmente, se encontró al cruzar la información de parentescos familiares con el porcentaje de participación de las familias, que la participación de ambos es de $50 \%$, dando una igualdad de participación a los géneros en caso de la herencia y ambos son propietarios igualitarios de la empresa que se constituye en estas condiciones. Un escaso porcentaje de EF se encuentra entre primos. De hecho, Miratex hizo parte de este pequeńo porcentaje. A pesar de prevalecer los lazos afectivos entre la familia, se halla una preferencia por los hermanos a la hora de constituir una empresa. La situación se explica por los encuestados porque en muchos de los casos son hijos únicos en la familia y prefieren asociarse con alguien de la familia antes que con un amigo, reforzando los lazos de confianza y afecto que existen entre sí, siempre y cuando los primos hayan tenido un crecimiento conjunto $y$ hayan compartido experiencias familiares que les permitan el fortalecimiento de esta condición de confianza, para tomar el riesgo de asumir la constitución de una empresa de este tipo.

Para el caso de la hipótesis alternativa en la cual se manifiesta que las EF dirigidas por sucesores que se han involucrado en su manejo desde temprana edad tienen más posibilidades de llevar a cabo un relevo generacional exitoso, se observó que, para el caso de Miratex, el sucesor se consideró administrativa y físicamente capaz de hacerse cargo de la propiedad, debido a su preparación académica y gerencial y a los años dedicados a la empresa. El predecesor todavía sigue asistiendo al trabajo diariamente y no ha renunciado a ninguna de sus responsabilidades, aunque, en la práctica, sean sus dos hijos los que dirigen la empresa de manera efectiva. El estilo que ha adoptado para su desvinculación de la empresa es el que se denominaría de embajador, según la clasificación de Sonnenfeld (1988), ya que, aunque no haya abandonado nunca su actividad en la empresa, esta actividad ha ido progresivamente limitándose al asesoramiento y apoyo al sucesor. 


\section{Conclusiones}

Un aspecto importante en el proceso de la sucesión es la transmisión de los puntos fundamentales de la cultura de la empresa a aquellas personas que la han de vivir y hacer evolucionar:

Para el caso de estudio, los factores relevantes para su proceso de sucesión se observan en la tabla 2 :

Tabla 2. Factores relevantes para su proceso de sucesión

\begin{tabular}{|c|c|}
\hline Factores objetivos & Factores subjetivos \\
\hline $\begin{array}{ll}\text { - } & \text { Posicionamien- } \\
\text { - } & \text { Permanencia } \\
\text { - } & \text { Ofrecimiento de } \\
\text { empleo }\end{array}$ & $\begin{array}{ll}\text { - } & \text { Relaciones interpersona- } \\
\text { - } \quad \text { les e intragrupales } \\
\text { Grado de satisfacción de } \\
\text { las necesidades de la fa- } \\
\text { milia } \\
\text { - Habilidades para resolver } \\
\text { - } \quad \text { problemas y amenazas } \\
\text { - } \quad \text { Políticas de dividendos } \\
\text { Disposición de incor- } \\
\text { porar familiares a la em- } \\
\text { presa }\end{array}$ \\
\hline
\end{tabular}

Por ello, es importante que el proceso de sucesión vaya trabajándose con anterioridad, ya que el aprendizaje en la empresa, por parte de los hijos, es importante en su formación como futuros directivos o propietarios. La capacitación del sucesor para desempeñarse en la gerencia de la empresa, la integración de los miembros de la familia propietaria y la planeación de la sucesión son factores, claramente, asociados con los resultados de la sucesión. Dentro de los desafíos más importantes a los que se enfrentan las empresas familiares en cualquier lugar del mundo, está el de la elección del sucesor. Del acierto en esta elección dependerá, en muchos casos, la supervivencia de la empresa. La literatura revisada en el presente artículo destaca algunos aspectos que son considerados como relevantes por la mayoría de los expertos. El criterio de elección del sucesor o el nivel de formación exigible son algunos de ellos. Tal como predecía el marco teórico desarrollado, ha sido contrastada la existencia de mayores niveles de compromiso en las EF que en las no familiares, concretado, a su vez, en la existencia de mayores niveles de identificación de lealtad y de implicación entre sus empleados. Los valores que se fomentan dentro de una familia varían de una a otro, por lo que estos deben ser aprovechados para darle fuerza a la familia frente a las variables que se manejan en la empresa; en este caso exitoso de sucesión, el predecesor no formó de una manera planificada al sucesor, más bien, le fueron asignadas responsabilidades, y lo que le permitió tomar decisiones según su criterio y que aprendiera de sus errores y sus aciertos. El predecesor, y también ciertos empleados que llevaban con él desde los comienzos de la empresa, estaban siempre disponibles para las consultas, y los conocimientos sobre el funcionamiento habitual de la empresa se transmitieron de una forma que es calificada como de completamente natural. De esta manera, el predecesor logró identificar habilidades potenciales que lo llevaron a tomar una decisión acertada en la escogencia del sucesor. En cuanto a las proposiciones relacionadas con el predecesor y el compromiso con el liderazgo familiar y con los descendientes específicos, la actitud por parte del predecesor que resulta más positiva es la de preferir la continuidad de la empresa como familiar, pero siempre que ello sea la opción más beneficiosa para la empresa. En todo caso y en consonancia con la prioridad de los fines de la empresa, la elección de 
un sucesor entre varios de los hijos es una decisión que se considera conveniente dejar a estos, de manera que ellos puedan decidir lo que se considera más oportuno para la empresa. Sin embargo, es de crucial importancia contar con la calificada opinión del predecesor, quien podrá identificar de manera más exacta el posible sucesor. La sucesión es un punto transcendental en el desarrollo de las empresas de familia, ya que, de no ser preparada con antelación, es el momento donde afloran los conflictos familiares internos que ponen en peligro la continuidad de la empresa.

\section{Limitaciones y futuras líneas de investigación}

Como toda línea de investigación, esta tiene debilidades que hacen, aún más, importante extenderla. La principal limitación es el acceso restringido a las empresas de familia, ya que por la misma protección de la información es difícil lograr ahondar en otros temas y, más aún, realizar cuadros comparativos de empresas del mismo sector.

Como futuras líneas que se desprenden de esta investigación están:

1) El análisis de las relaciones existentes entre el sector productivo (textil) y la tendencia de factores para el relevo generacional.

2) La importancia del protocolo familiar o el mecanismo más acertado para la sucesión.

3) Encontrar si las empresas familiares tienen mayor permanencia en el mercado que las empresas no familiares.

4) Cómo afectará la visión patrimonial el futuro del grupo familiar, la estabilidad de vínculos y la estabilidad económica.

\section{Referencias}

I. Aronoff, C. y J. Ward (1994), Set policies to solve future problems. Nation's Business, 82, 70-71.

2. Aronoff, C. y Ward, J. (1991). Family business sourcebook. Míchigan: Omnigraphics.

3. Astrachan J. y Kolenko T. (1994). A neglected factor explaining family business success: human resource practices. Family Business Review, 7(3), 251-262.

4. Avolio, B. J. y Bass, B. M. (1991). Fullrange training of leadership. Binghamton: Bass/Avolio and Associates.

5. Barach, J. A. y Ganitsky, J. B. (1995). Successful succession in family business. Family Business Review, 8, 131155.

6. Barach, J. A.; Ganitsky, J. B.; Carson, J. A. y Doochin, B. A. (1988). Entry of the next generation strategic challenge for family business. Journal of Small Business Management, 26, 49-56.

7. Barnes, L. B. y Hershon, S. A. (1976). Transferring power in family business. Harvard Business Review, 54, 105-114.

8. Bass, B. M. (1998). Transformational leadership: industrial military and educational impact. Mahwah: Lawrence Elbaum Associates Publishers.

9. Bass, B. M. (1999). Two decades of research and development in transformational leadership. European Journal of Work and Organizational Psychology, 8, 9-32.

Iо. Bass, B. M. y Steidlmeier, P. (1999). Ethics, character and authentic transformational leadership behavior. Leadership Quarterly, 10, 181-217. 
I I. Beckhard, R., y Harris, R. (1988). Organizational transitions (2. ${ }^{\mathrm{a}} \mathrm{ed}$.). Addison-Wesley Iberoamericana.

I 2. Beckhard, R., \& Dyer Jr, G. (1983). Managing continuity in the familyowned business. Organizational Dynamics, 12(1), 5-12.

I 3. Belausteguigoitia, I. (2004). Empresas familiares: su dinámica, equilibrio y consolidación. México: McGraw-Hill.

I4. Birley, S. (1986). Succession in the family firm: the inheritor view. Journal of Small Business Management, 24, 36-43.

I 5. Casrud, A. y Brannback, M. (2007). Entrepreneurship. Westport (Connecticut): Greenwood Publishing Group, Inc.

I6. Chami, R. (1999). What's different about family businesses? University of Notre Dame Working Paper. Notre Dame: University of Notre Dame.

I7. Churchill, N. C. y Hatten, K. J. (1987). Non-market-based transfer of wealth and power: a research framework for family businesses. American Journal of Small Business, 12, 53-66.

I 8. Daily, C. y Dollinger, M. (1991). Family Firms are differents. Review of Business (13), 3-5.

I9. Danco, K. (1981). From the other side of the bed. Ohio: The Center for Family Business.

20. Danco, L. A. (1980). Inside the family business. Ohio: The Center for Family Business.

2 I. Danco, L. A. (1992). Beyond survival. Ohio: The Center for Family Business.

22. Davis, E. (1992). Long live the business. Small Business Reports, 17(11), 30-40.

23. DiMaggio, P. y Powell, W. (1983). The iron cage revisited: institutional isomorphism and collective rationality in organizacional fields. American Sociological Review, 147.

24. Domínguez, P. (2010). Sucesión en las empresas familiares. Lima: PAD/Escuela de Dirección de la Universidad de Piura.

25. Dumas, C.; Dupuis, J. P.; Richer, F y St-Cyr, L. (1995). Factors that influence the next generation's decision to take over the family farm. Family Business Review, 8, 99-120.

26. Dunn, B. (1995). Success themes in scottish family enterprises: philosophies and practices through the generations. Family Business Review, 8, 17-28.

27. Dyck, B. y Mauws, M. (2002). Passing the baton: the importance of sequence, timing, technique, and communication in executive succession. Journal of Business Venturing, 17, 43-62.

28. Dyer, W. J. (1994). Potential contributions of organizational behavior to the study of family-owned businesses. Family Business Review (7), 2, 109-131.

29. Dyer, W. G., Jr. (1986). Cultural change in family firms: anticipating and managing business and family transitions. San Francisco, CA: Jossey-Bass.

30. Elstrodt, H. (2003). Keeping the family in business (Elstrodt es director de la oficina McKinsey o Company). Recuperado de http://portal.fedepalma.org// noticias/156.htm

31. Fiegener, M. y Brown, B. (1994). A comparison of successor development in family and non-family businesses. Family Business Review, 7(4), 313-329.

32. File, K. y Prince, R. (1996). Attribution for family business failures: the heir's perspective. Family Business Review, 9(2), 171-184.

33. Fiol, C.; Harris, D. y R. House (1999). Charismatic leadership: strategies for 
effecting social change. Leadership Quarterly, 10, 449-482.

34. Friedman, S. (1986). Succession systems in large corporations: characteristics and correlations of performance. Human Resource Management, 25, 191213.

35. Friedman, S. (1991). Siblings relationships and intergenerational succession in family firms. Family Business Review, 4, 3-20.

36. Friskoff, P. A. y Brown, B. M. (1993). Women on the move in family business. Business Horizons, 36, 66-70.

37. Fundes (2008). Dinámica de la empresa familiar. Bogotá: Fundes International.

38. Gallo, M. A. (1997). La empresa familiar. Pamplona: IESE/Universidad de Navarra.

39. Gersick, K. E.; Davis, J.; McCollom, M. y Lansberg, I. (1997). Generation to Generation: life cicles of the family business. Harvard: Harvard Business School Press.

40. Goldberg, S. D. (1996). Research note: effective successors in family-owned businesses: significant elements. Family Business Review, 9(2), 185-197.

4I. Goldberg, S. D. (1991). Factors which impact effective succession in small family-owned businesses: an empirical study. UMI Dissertation Services.

42. Gómez-Betancourt, G. (2005). ¿Son iguales todas las empresas familiares? Barcelona: Granica.

43. González, F.; Guzmán, A.; Trujillo M. (2010, enero-abril). The role of heirs in familiy businesses: the case of Carvajal. Innovar Journal, 20(36), 49-65.

44. Handler, W. C. (1989). Methodological issues and considerations in studying family businesses. Family Business Review, 2(3), 257-376.
45. Handler, W. C. (1989). Managing the family firm succession process: the next-generation family member's experience. UMI Dissertation Services.

46. Ibrahim, A. y Dumas, C. (2001). Strategies decision making in small family firms: an empirical investigation. Journal of Small Business Strategy, 12(1), 1-11.

47. Instituto de Estudios Superiores de la Empresa (IESE) (1986). La empresa familiar. Barcelona: Editorial Vicente Font Pascual.

48. Jaramillo, O. (2003). Gestión del talento humano en la micro, pequeña y mediana empresa vinculada al programa Expopyme de la Universidad del Norte en los sectores de confecciones y alimentos. Recuperado de http:// ciruelo. uninorte.edu.co/pdf/pensamiento_gestion/18/4_Gestion\%20del\%20talento\%20humano.pdf

49. Kark, R.; Shamir, B. y Gilad, C. (2003). The two faces of transformational leadership: empowerment and dependency. Journal of Applied Psychology, 88, 246-255.

50. Kesner, I. F. y Sebora, T. C. (1994). Executive succession: past, present $\&$ future. Journal of Management, 20, 327372.

5I. Kets de Vries, M. (1993). The dynamics of family-controlled flrms: the good and bad news. Organizational Dynamics, 21, 59-71.

52. Lansberg, I. S. (1989). The succession conspiracy: resistance to succession planning in first generation family firms. Family Business Review, 1, 119143.

53. Lea, J. (1993). La sucesión del management. Buenos Aires: Granica. 
54. Lee, J. (2006). Family firm performance: further evidence. Family Business Review, 19, 103-114.

55. Levinson, H. (1981). Conflict that plague family business. Family Business Review, 49, 90-98.

56. Longenecker, J. G. y Schoen, J. E. (1978). Management succession in the family business. Journal of Small Business Management, 16, 1-6.

57. Montoya, A. y Montoya, I. (2010, 2528 de junio). El estudio de casos como una posibilidad de desarrollo de competencias gerenciales en la enseñanza de administración de empresas. En: WACRA $^{\star} 2010$, the 27th International Conference on Case Method Research and Application, Barranquilla, Colombia.

58. Morris, M.; Williams, R. y Allen, J. (1997). Correlates of success in family business transitions. Journal of Business Venturing, 12(5), 385-401. Recuperado de

59. http://www.navactiva.com/es/actualidad/el-67-de-las-empresas-familiarescierra-con-el-cambio-generacion_6114

6o. Nelton, S. (1991). When widows take charge. En Aronoff, C. E. y Ward, J. L. (eds.). Family business sourcebook. Míchigan: Omnigraphics, Inc.

6I. Poza, M. E. (1989). Smart growth: critical choices for business continuity and prosperity. California: Jossey-Bass Inc., Publishers.

62. Poza, M. E. (1995). A la sombra del roble: la empresa privada familiar y su continuidad. Ohio: Editorial Universitaria para la Empresa Familiar.

63. Robinson, K. B., Jr. (1982). The importance of outsiders in small firms strategic planning. Academy of Management Journal, 25, 90-93.
64. Romero, L. E. (2006). Competitividad y productividad en empresas familiares pymes. Revista EAN, 57, 131-142.

65. Rosenblatt, P. C.; Mik, L. de; Anderson, R. M. y Johnson, P. A. (1985). The family in business. California: JosseyBass Inc.

66. Schulze, W.; Lubatkin, M.; Dino, R. y Buchholtz, A. (2001). Agency relationships in family firms: theory and evidence. Organization Science, 12(2), 99116

67. Scott, W. (1992). Organizations: rational, natural and open systems. Nueva York: Prentice Hall.

68. Shanker, M. C. y Astrachan, J. H. (1996). Myths and realities: family businesses' contribution to the US economy: a framework for assessing family business statistics. Family Business Review, 9, 107-123.

69. Sharma, P.; Chrisman, J. J. y Chua, J. H. (1996). A review and annotated bibliography of family business studies. Norwell, MA: Kluwer Academic Publishers.

70. Sharma, P.; Chrisman, J. J.; Pablo, A. y Chua, J. H. (2001). Determinants of initial satisfaction with the succession process in family firms: a conceptual model. Entrepreneurship Theory and Practice, 25(3), 1-19.

7r. Shulman, M. G. (1991, julio). Successful succession planning, CA Magazine, 124(7), 43-50.

72. Socorro, F. (2008). Empresas familiares y empresas de familia: ¿conoce la diferencia? Recuperado de http//:www. degerencia.com

73. Sonnenfeld, J. (1988). The hero's farewell: what happens when CEOs retire? Nueva York.

74. Oxford University Press. 
75. Sosik, J.; Potosky, D. y Jung, D. (2002). Adaptive self-regulation: meetings others' expectations of leadership and performance. The Journal of Social Psychology, 142, 211-232.

76. Stempler, G. L. (1988). A study of succession in family owned businesses. UMI Dissertation Services.

77. Superintendencia de Sociedades (2006). Boletín Estadistico. Bogotá: Superintendencia de Sociedades

78. Swogger, G., Jr. (1991). Assessing the successor generation in family business. Family Business Review, 4(4), 397-411

79. Vélez, D. (2005). Cómo gerenciar bien empresas de familia. Forum. Bogotá: Universidad de La Sabana.

80. Viator, R. (2001). The relevance of transformational leadership to nontraditional accounting services: information systems assurance and business consulting. Journal of Information Systems, 15, 99-125.
81. Walumbwa, F.; Lawler, J.; Avolio, B.; Wang, P. y Shi, K. (2005). Transformational leadership and work-related attitudes: the moderating effects of collective and self-efficacy across cultures. Journal of Leadership and Organizational Studies, 11, 2-16.

82. Ward, J. L. (1987). Keeping the family business healthy. San Francisco, CA: Jossey-Bass.

83. Wharton Entrepreneurial Center (1984). A unified systems theory of family firm performance, Wharton School Working Paper Series, University of Pennsylvania.

84. Yukl, G. (2002). Leadership in organizations (5. ${ }^{a}$ ed.). Upper Saddle River: Prentice-Hall. Recuperado de http:// www.acc10.cat/ACC1O/cat/binaris/ Esm_Feb_07_FBK_tcm176-79576. pdf 